\title{
Orbits of Curves on Certain K3 Surfaces
}

\author{
ARTHUR BARAGAR \\ Department of Mathematical Science, University of Nevada, Box 454020, 4505 Maryland \\ Parkway, Las Vegas, NV 89154-4020,U.S.A.e-mail: baragar@unlv.edu
}

(Received: 31 October 2000; accepted in final form: 28 March 2002)

Abstract. In this paper, we study the family of algebraic K 3 surfaces generated by the smooth intersection of a $(1,1)$ form and a $(2,2)$ form in $\mathrm{P}^{2} \times \mathrm{P}^{2}$ defined over $\mathrm{C}$ and with Picard number 3. We describe the group of automorphisms $\mathcal{A}=\operatorname{Aut}(V / \mathrm{C})$ on $V$. For an ample divisor $D$ and an arbitrary curve $C_{0}$ on $V$, we investigate the asymptotic behavior of the quantity $N_{\mathcal{A}\left(C_{0}\right)}(t)=\#\left\{C \in \mathcal{A}\left(C_{0}\right): C \cdot D<t\right\}$. We show that the limit

$$
\lim _{t \rightarrow \infty} \frac{\log N_{\mathcal{A}(C)}(t)}{\log t}=\alpha
$$

exists, does not depend on the choice of curve $C$ or ample divisor $D$, and that $.6515<\alpha<.6538$.

Mathematics Subject Classifications (2000). 14J28, 14J50, 14G05, 14H37, 11D41, $11 \mathrm{D} 72$.

Key words. fractal dimension, K3 surface, Picard group.

\section{Introduction}

Let $V$ be an algebraic $\mathrm{K} 3$ surface defined over a field $K \subset \mathrm{C}$ and let $\mathcal{A}=\operatorname{Aut}(V / K)$ be its group of automorphisms. Let $D$ be an ample divisor in $\operatorname{Pic}(V)$ and let $C_{0}$ be a curve on $V$. Define the quantity

$$
N_{\mathcal{A}\left(C_{0}\right)}(t)=N_{\mathcal{A}\left(C_{0}\right)}(t, D)=\#\left\{C \in \mathcal{A}\left(C_{0}\right): D \cdot C<t\right\} .
$$

This quantity has been studied for several classes of K3 surfaces. If $\mathcal{A}$ is finite (for example, if the Picard number for $V$ is one, or if the Picard number is two and $V$ contains a curve with self intersection 0 or -2$)$, then $N_{\mathcal{A}(C)}(t)$ is trivially bounded for all $t$. If $V$ has Picard number two, has no curves with self intersection equal to 0 or -2 , and $K$ is sufficiently large, then $N_{\mathcal{A}(C)}(t) \gg \ll \log (t)$ [1]. For the family of $\mathrm{K} 3$ surfaces studied in [2, 4], and [7] (which have Picard number 3 and no -2 curves), we have $N_{\mathcal{A}(C)}(t) \gg \ll t$. Similar quantities have been studied by Silverman [19] and Billard [7]. Such studies are motivated, in part, by the conjectures of Batyrev and Manin on the distribution of rational points on arbitrary varieties [6].

In this paper, we study the family of K3 surfaces generated by the smooth intersection of a $(1,1)$ form and a $(2,2)$ form in $\mathbb{P}^{2} \times \mathbb{P}^{2}$, which is sometimes called the family of Wehler K3 surfaces, named after the researcher who first studied 
them [20]. The family of Wehler K3 surfaces has an eighteen dimensional moduli space. The generic Wehler surface has Picard number 2 and no -2 curves. In this paper, we study the class of Wehler K3 surfaces with Picard number 3. In Section 3 , we show the following main result:

THEOREM 0.1. Let $V$ be a Wehler $\mathrm{K} 3$ surface defined over $\mathrm{C}$ and with Picard number 3. Let $D$ be an ample divisor and let $C$ be a curve on $V$. Then, the limit

$$
\lim _{t \rightarrow \infty} \frac{\log \left(N_{\mathcal{A}(C)}(t)\right)}{\log t}=\alpha
$$

exists and does not depend on the curve $C$ or ample divisor D. Furthermore, $.6515<\alpha<.6538$.

To study $N_{\mathcal{A}(C)}(t)$, we must first be familiar with the group $\mathcal{A}$. There exists a natural map (the pullback map) from $\mathcal{A}$ into the group of automorphisms of the lattice $\operatorname{Pic}(V)$. In [16], Pjatecki1̌-S̆apiro and S̆afarevič show that this map has a finite kernel, and describe how to find a subgroup $\mathcal{O}^{\prime \prime}$ of this group such that the pullback map sends $\mathcal{A}$ into $\mathcal{O}^{\prime \prime}$ and has a finite cokernel. The main result of Section 2 is an application of this fundamental result to our particular case:

THEOREM 0.2. Let $V$ be a Wehler $\mathrm{K} 3$ surface defined over $\mathrm{C}$ and with Picard number 3 . Then the group $\mathcal{O}^{\prime \prime}$ is generated by linear maps $T_{1}, T_{2}$, and $T_{3}$ where, in a suitable basis of $\operatorname{Pic}(V) \otimes Q$ (to be described in Section 2), we have

$$
T_{1}=\left[\begin{array}{ccc}
0 & 0 & 1 \\
3 & -1 & 3 \\
1 & 0 & 0
\end{array}\right], \quad T_{2}=\left[\begin{array}{ccc}
-1 & 4 & 1 \\
0 & 1 & 0 \\
0 & 0 & 1
\end{array}\right], \quad \text { and } \quad T_{3}=\left[\begin{array}{ccc}
1 & 0 & 0 \\
0 & 1 & 0 \\
4 & 14 & -1
\end{array}\right] .
$$

Furthermore, $\mathcal{O}^{\prime \prime} \cong Z / 2 Z * Z / 2 Z * Z / 2 Z$.

Hence, the problem of finding $\alpha$ can be reduced to a problem in linear algebra. This is not to say it becomes trivial. In fact, $\alpha$ can be thought of as the dimension of a fractal that has no self similarity. The techniques we use to calculate $\alpha$ are along the lines of those used by Boyd [8-10] to calculate the fractal dimension of the residual set in the Apollonian packing.

In Section 1, we also point out the following general result:

THEOREM 0.3. Suppose $V$ is a $\mathrm{K} 3$ surface defined over a number field $K($ or $\mathrm{C})$ and with Picard number $n \geqslant 3$. Let $C$ be a curve on $V$ with nonnegative self intersection $k$. Then $N_{\mathcal{A}(C)}(t) \ll t^{n-2}$.

This result is essentially due to Pjateckiı̌-S̆apiro and S̆afarevič [16] and Lax and Phillips [14]. The author's contribution is mostly one of observation.

Our interest in $N_{\mathcal{A}(C)}(t)$ is in part motivated by its presumed relation to the more arithmetic quantity $N_{\mathcal{A}(P)}(t)$ for a rational point $P$ in a number field $K$, using a Weil 
height $h_{D}$ where $D$ is the same ample divisor. Such quantities have been studied by Silverman [19] for Wehler K3 surfaces with Picard number two, and the author [2] for a class of K3 surfaces with Picard number three and no -2 curves. It is known that

$$
N_{\mathcal{A}(P)}(t) \gg \ll N_{\mathcal{A}(C)}(t)
$$

for generic rational points $P$ on K3 surfaces with Picard number 2 [1] and the K3 surfaces with Picard number three studied in [2] and [4]. The result is vacuously true for K 3 surfaces with Picard number one, since in that case, $\mathcal{A}$ is finite. It would not surprise me if this relation is true in general. We will elaborate on this thought at the end of the paper.

\section{Background}

Let $V$ be an algebraic $\mathrm{K} 3$ surface defined over $K \subset \mathrm{C}$. That is, $V$ is a surface with trivial canonical divisor and irregularity equal to zero. If $C$ is a smooth curve on $V$, then the genus of $C$ is given by the adjunction formula $2 g-2=C \cdot C$. The arithmetic genus $\rho_{a}$ of $V$ is 1 , so the Riemann-Roch formula on $V$ is

$$
l(D)+l(-D) \geqslant(1 / 2) D \cdot D+2 .
$$

Our notation is as in Hartshorne [11]. If $l(D)>0$ for some divisor $D$, then $D$ is effective. Thus, for any divisor $D$ with $D \cdot D \geqslant-2$, either $D$ or $-D$ is effective. The Picard group $\operatorname{Pic}(V)$ is a lattice of dimension $n \leqslant 20$. The dimension $n$ is called the Picard number. Let $\mathcal{D}=\left\{D_{1}, \ldots, D_{n}\right\}$ be a basis of $\operatorname{Pic}(V)$, so

$$
\operatorname{Pic}(V)=\left\{a_{1} D_{1}+\cdots+a_{n} D_{n}: a_{k} \in Z \text { Z }\right\} .
$$

Let $Q=\left[D_{i} \cdot D_{j}\right]$ be the intersection matrix for $V$ with respect to the basis $\mathcal{D}$. By the Hodge index theorem, the signature of $Q$ is $(1, n-1)$. That is, $Q$ has one positive eigenvalue and $n-1$ negative eigenvalues.

Let $\mathcal{A}=\operatorname{Aut}(V / K)$ be the group of automorphisms on $V$. For an automorphism $\sigma \in \mathcal{A}$, the pullback $\sigma^{*}$ acts linearly on $\operatorname{Pic}(V)$. Since $\sigma$ preserves intersections, we further have that $\sigma^{*}$ is in

$$
\mathcal{O}=\mathcal{O}(Z)=\left\{T \in M_{n \times n}(Z): T^{T} Q T=Q\right\} .
$$

For an ample divisor $D$, the hypersurface $\mathbf{x}^{T} Q \mathbf{x}=D \cdot D$ is a hyperboloid of two sheets, one of which contains $D$. Let us distinguish this sheet with $\mathcal{H}$, and define

$$
\mathcal{O}^{+}=\mathcal{O}^{+}(Z)=\{T \in \mathcal{O}: T(\mathcal{H})=\mathcal{H}\}
$$

The surface $\mathcal{H}$ can be thought of as a model of $n$-1-dimensional hyperbolic geometry imbedded in a Lorentz space, where the Lorentz inner product is the negative of the intersection product. The distance $|A B|$ between two points $A$ and $B$ in this model $\mathcal{H}$ is given by

$$
A^{T} Q B=A \cdot B=\| A|||| B|| \cosh (|A B|)=-D \cdot D \cosh (|A B|),
$$


where $\|A\|=\sqrt{-A \cdot A}$. With this metric, the group of isometries on $\mathcal{H}$ is $\mathcal{O}^{+}(\mathbb{R})$, where the definition of this group is the obvious analog of $\mathcal{O}^{+}(Z)$. For more details of this model, we refer the reader to Ratcliffe [18] or a more elementary treatment by the author in [5].

Let $\mathcal{E}$ be the set of effective divisor classes in $\operatorname{Pic}(V)$. That is, $E \in \mathcal{E}$ if we can write $E=a_{1} C_{1}+\cdots+a_{m} C_{m}$ with $a_{i} \geqslant 0$ and $C_{i}$ a divisor class that can be represented with a curve in $V$. Let

$$
W=\{C \in \operatorname{Pic}(V): C \cdot C \geqslant 0, C \cdot E \geqslant 0 \text { for all } \mathrm{E} \in \mathcal{E}\} .
$$

It is clear that if $\sigma \in \mathcal{A}$, then $\sigma^{*}(W)=W$, so let us define

$$
\mathcal{O}^{\prime \prime}=\left\{T \in \mathcal{O}^{+}: T W=W\right\} .
$$

If there are any -2 curves on $V$, then there exists a large subset of $\mathcal{O}^{+}$that cannot be in $\mathcal{O}^{\prime \prime}$. For an element $C \in \operatorname{Pic}(V)$ such that $C \cdot C=-2$, let us define the reflection through $C$ by $R_{C} D=D+(C \cdot D) C$. Note that $R_{C}$ is in $\mathcal{O}$, since it preserves intersections. However, by the Riemann-Roch theorem, either $C$ or $-C$ is effective. Hence, since $R_{C} C=-C$, we have that $R_{C} \notin \mathcal{O}^{\prime \prime}$. Let $\mathcal{O}^{\prime}$ be the subgroup of $\mathcal{O}$ generated by the reflections through -2 curves. Note that $T R_{C} T^{-1}=R_{T C}$. Hence, $\mathcal{O}^{\prime}$ is a normal subgroup of $\mathcal{O}$. In [16], Pjateckii-Šapiro and $\breve{S}$ afarevič show that the natural map

$$
\begin{aligned}
\Phi: \operatorname{Aut}(V / \mathrm{C}) & \rightarrow \mathcal{O}^{\prime \prime} \\
\sigma & \mapsto \sigma^{*}
\end{aligned}
$$

has a finite kernel and co-kernel, and that $\mathcal{O}^{\prime \prime} \cong \mathcal{O}^{+} / \mathcal{O}^{\prime}$.

As a corollary, we get the following rather nice and general result which, as far as I know, has not been expressed in print.

THEOREM 1.1. Suppose $V$ is an algebraic $\mathrm{K} 3$ surface defined over a field $K \subset \mathbb{C}$ and with Picard number $n \geqslant 3$. Let $C$ be a curve on $V$ with nonnegative self intersection $k$. Then $N_{\mathcal{A}(C)}(t) \ll t^{n-2}$.

Proof. By the result of Pjatecki1-S̆apiro and S̆afarevič, we know that $\Phi$ has a finite kernel. Thus, for any $K \subset \mathbb{C}$, we know $N_{\mathcal{A}(C)}(t) \ll N_{\mathcal{O}^{+}(C)}(t)$, where we have abused notation a little by letting $C$ represent both the curve and the divisor class it represents in $\operatorname{Pic}(V)$. Recall that, if $k>0$, then the surface $\left\{\mathbf{x} \in \mathbb{R}^{n}: \mathbf{x}^{T} Q \mathbf{x}=k\right\}$ is a (hyper)hyperboloid of two sheets and the sheet $\mathcal{H}$ that contains $C$ is an $n$-1-dimensional hyperbolic space. (To avoid overusing the prefix 'hyper-', in the rest of this proof we will use the appropriate terminology for $n=4$.) The group $\mathcal{O}^{+}$is a discrete subgroup of the group of isometries on $\mathcal{H}$. If $D$ is ample, then in particular $D \cdot D>0$, so there exists a constant $\lambda=k / \sqrt{D \cdot D}$ such that $\lambda D \in \mathcal{H}$. The surface in $\mathcal{H}$ described by the equation $D \cdot \mathbf{x}=t$ is a sphere of hyperbolic radius $r=\log (t)+\mathrm{O}(1)$. To see this, we note that

$$
D \cdot \mathbf{x}=\frac{1}{\lambda}(\lambda D) \cdot \mathbf{x}=\frac{k}{\lambda} \cosh \left(\left|D^{\prime} X\right|\right),
$$


where $D^{\prime}$ and $X$ are the points in $\mathcal{H}$ represented by $\lambda D$ and $\mathbf{x}$. Thus,

$$
\left|D^{\prime} X\right|=\operatorname{arccosh}(\lambda t / k)=\log (t)+\mathrm{O}(1) .
$$

In particular, this distance is constant so we get a sphere. Thus, our problem now is to count the number of lattice points in a ball of radius $r$. In Euclidean geometry, such problems have almost trivial geometric solutions. In hyperbolic geometry, the geometric arguments do not work, since the volume of a ball and the surface area of its boundary have the same order of magnitude. However, using analytic methods, Lax and Phillips [14] show that, with reasonable assumptions, the expected indeed occurs. Precisely, for a lattice with a fundamental domain that has finite volume and is bounded by a finite number of planes, they show that the number of lattice points in a ball of radius $r$ grows asymptotically like the volume of the ball divided by the volume of the fundamental domain of the lattice. It is known that arithmetic groups (like $\mathcal{O}^{+}$) have such fundamental domains [17]. Thus,

$$
N_{\mathcal{A}(C)}(t) \ll N_{\mathcal{O}^{+}(C)}(t) \gg \ll \mathrm{e}^{r(n-2)} \gg \ll t^{n-2} .
$$

Furthermore, $N_{\mathcal{A}(C)}(t) \gg t^{n-2}$ if $V$ contains no -2 curves and $K$ is sufficiently large.

Suppose now that $k=0$. Then the equation $\mathbf{x}^{T} Q \mathbf{x}=0$ is homogeneous and describes an $n$-2-dimensional ellipsoid in $\mathbb{P}^{n-1}$. If $d$ is the greatest common factor of the components of $C$ and $\sigma^{*} \in \mathcal{O}^{\prime \prime}$, then $d$ divides all components of $\sigma^{*} C$ since $\sigma^{*}$ is linear and has integer entries. Since $\sigma_{*}=\left(\sigma^{*}\right)^{-1}$ is in $\mathcal{O}^{\prime \prime}$ and also has integer entries, $d$ must be the greatest common factor of $\sigma^{*} C$. Thus every element of $\mathcal{O}^{\prime \prime}(C)$ can be thought of as a rational point on this ellipsoid. Furthermore, the function $H(\mathbf{x})=D \cdot \mathbf{x}$ is an exponential height on the ellipsoid. Finally, it is well known that the number of rational points on an ellipsoid of dimension $n-2$ with exponential height bounded by $t$ is $\mathrm{O}\left(t^{n-2}\right)$. Since $\mathcal{O}^{\prime \prime}(C)$ is a subset of these points, we have $N_{\mathcal{A}(C)}(t) \ll t^{n-2}$.

\section{Wehler K3 Surfaces}

Let $V$ be the smooth intersection of a $(1,1)$ form and a $(2,2)$ form in $\mathrm{P}^{2} \times \mathrm{P}^{2}$. In [20], Wehler shows that such a surface is a K3 surface.

Let $(X, Y) \in \mathbb{P}^{2} \times \mathbb{P}^{2}$, and let $X=\left(X_{0}, X_{1}, X_{2}\right)$. We can write the $(1,1)$ form as

$$
\sum_{i=0}^{2} F_{i}(Y) X_{i}=0
$$

where $F_{i}(Y)$ is linear in $Y$. We can similarly write the $(2,2)$ form as

$$
\sum_{0 \leqslant i \leqslant j \leqslant 2} G_{i j}(Y) X_{i} X_{j}=0,
$$

where $G_{i j}(Y)$ is quadratic in $Y$. Let $\pi_{1}$ and $\pi_{2}$ be the projections of $V$ onto, respectively, the first and second copies of $\mathbb{P}^{2}$. Let us fix $Y \in \mathbb{P}^{2}$. From (1), we can solve for one of the $X_{i}$ in terms of $X_{j}$ and $X_{k}$. Plugging this into (2), we get a quadratic 
in $X_{j}$ and $X_{k}$ that has coefficients that are functions of $Y$. Hence, if these coefficients are not all zero, then $\pi_{2}^{-1}(Y)$ is exactly two points (counting multiplicity). The coefficients are all zero for at most a finite number of points, for otherwise, $V$ would be singular. Hence, $\pi_{2}$ is a double cover of $V$ over $\mathrm{P}^{2}$ at all but a finite set of points.

In this class of K3 surfaces, the generic surfaces $V$ were studied by Wehler [20]. In this case, both $\pi_{1}$ and $\pi_{2}$ are everywhere double covers; the Picard number for $V$ is two; and $V$ contains no -2 curves. If $H_{1}$ and $H_{2}$ are hyperplane sections in respectively the first and second copies of $\mathbb{P}^{2}$, then $\left\{D_{1}, D_{2}\right\}$ is a basis for $\operatorname{Pic}(V)$ where $D_{i}=\pi_{i}^{*} H_{i}$. The intersection matrix is

$$
Q=\left[D_{i} \cdot D_{j}\right]=\left[\begin{array}{ll}
2 & 4 \\
4 & 2
\end{array}\right] .
$$

In this paper, we study the Wehler K3 surfaces with Picard number three. These are the varieties for which (say) $\pi_{1}$ is everywhere a double cover, but for which there exists exactly one point $P \in \mathbb{P}^{2}$ such that $\pi_{2}^{-1}(P)$ is more than two points. Then $\pi_{2}^{-1}(P)$ is the set of points $(X, P)$ which lie on the $(1,1)$ form. That is, $\pi_{2}^{-1}(P)$ is a line in $V$. In particular, it is a smooth rational curve and, hence, is a -2 curve in $V$ (by the adjunction formula).

Such surfaces are plentiful, since their moduli space is 17-dimensional. For example, the $(2,2)$ form

$$
\begin{gathered}
\left(X_{0} X_{1}+X_{2}^{2}\right) Y_{0}^{2}+\left(X_{0} X_{2}+X_{1}^{2}\right) Y_{1}^{2}+\left(X_{0}^{2}+X_{1}^{2}-X_{2}^{2}\right) Y_{0} Y_{2}+ \\
+\left(X_{0}^{2}-X_{1} X_{2}\right) Y_{0} Y_{1}+\left(X_{0}^{2}-X_{1}^{2}+X_{2}^{2}\right) Y_{1} Y_{2}=0
\end{gathered}
$$

includes all points $(X, P)$ where $P=(0,0,1)$. Any $(1,1)$ form whose intersection with $(X, P)$ is a line and that intersects this $(2,2)$ form smoothly yields a Wehler $\mathrm{K} 3$ surface with Picard number three.

As before, let $D_{i}=\pi_{i}^{*} H_{i}$ for $i=1$ and 2. Let $D_{3}=\pi_{2}^{-1}(P)$. The calculations for the intersections of $D_{1}$ and $D_{2}$ with each other and with themselves are no different than in [20] and [19]. Note that $D_{2} \cdot D_{3}=0$, since if $H_{2}$ does not contain $P$, then $\pi_{2}^{-1} H_{2}$ and $\pi_{2}^{-1}(P)$ are disjoint. As noted before, a point is on $D_{3}$ if it has the form $(X, P)$ and lies on the $(1,1)$ form. Therefore, the projection $\pi_{1}\left(D_{3}\right)$ of $D_{3}$ onto $\mathbb{P}^{2}$ is a line $L$, and this projection is one-to-one. Thus, $D_{1} \cdot D_{3}=H_{1} \cdot L=1$. The intersection matrix with respect to the basis $\mathcal{D}=\left\{D_{1}, D_{2}, D_{3}\right\}$ is therefore

$$
Q_{\mathcal{D}}=\left[D_{i} \cdot D_{j}\right]=\left[\begin{array}{ccc}
2 & 4 & 1 \\
4 & 2 & 0 \\
1 & 0 & -2
\end{array}\right] .
$$

We include the subscript $\mathcal{D}$ on $Q$ to emphasize the basis used, since in the course of this paper, we will change the basis a couple of times.

For a point $(X, Y)$ on $V$, let $\pi_{1}^{-1}(X)=\left\{(X, Y),\left(X, Y^{\prime}\right)\right\}\left(\pi_{1}\right.$ is everywhere a double cover), and define $\sigma_{1}(X, Y)=\left(X, Y^{\prime}\right)$. Since $Y$ and $Y^{\prime}$ are roots of a quadratic, we can write $Y^{\prime}$ as a rational function in $X$ and $Y$ in a neighborhood of this point. Since 
this can be done for any point in $V$, the map $\sigma_{1}$ is a morphism, and since $\sigma_{1}^{2}$ is the identity, $\sigma_{1}$ is an automorphism of $V$.

We can similarly define $\sigma_{2}$ by $\sigma_{2}(X, Y)=\left(X^{\prime}, Y\right)$, for $Y \neq P$. We can extend $\sigma_{2}$ to $D_{3}$ in a natural way. This is done by first solving for (say) $X_{0}$ in (1) and plugging into (2) to get a quadratic equation of the form $A_{11}(Y) X_{1}^{2}+A_{12}(Y) X_{1} X_{2}+A_{22}(Y) X_{2}^{2}=0$. Let $P=\left(P_{0}, P_{1}, P_{2}\right)$. Without loss of generality, we may assume $P_{0} \neq 0$. Hence, we can consider an affine neighborhood of $P$ by setting $\left(y_{1}, y_{2}\right)=\left(Y_{1} / Y_{0}, Y_{2} / Y_{0}\right)$. Let us also write $\left(p_{1}, p_{2}\right)=\left(P_{1} / P_{0}, P_{2} / P_{0}\right)$. Let us set $S_{0} y_{1}=S_{1} y_{2}$. Then, we can think of $A_{i j}(Y)$ as functions of $y_{1}$ and $S \in \mathbb{P}^{1}$. Let us write $a_{i j}\left(y_{1}, S\right)=$ $A_{i j}\left(S_{1}, S_{1} y_{1}, S_{0} y_{1}\right)$. Note that $a_{i j}\left(p_{1}, S\right)=0$, so $\left(y_{1}-p_{1}\right)$ divides $a_{i j}$ for all $i$ and $j$. Thus, we can divide through by this factor, which gives us a quadratic with the same solutions $\left(X_{1}, X_{2}\right)$ for all $Y \neq P$ and in a neighborhood of $P$. But the coefficients of this equation are not all zero when $Y=P$. Thus, we can extend $\sigma_{2}$ to $D_{3}$. This extended map is a morphism of $V$ and hence, $\sigma_{2}$ is in $\mathcal{A}$.

Let us now calculate $\sigma_{2 *}=\left(\sigma_{2}^{-1}\right)^{*}$. Since $\sigma_{2}$ is an involution, $\sigma_{2 *}=\sigma_{2}^{*}$. Thus, $\sigma_{2 *} D_{i} \cdot D_{j}=D_{i} \cdot \sigma_{2}^{*} D_{j}=D_{i} \cdot \sigma_{2 *} D_{j}$. Note that $\sigma_{2 *} D_{2}=D_{2}$ and $\sigma_{2 *} D_{3}=D_{3}$. Thus,

$$
\begin{aligned}
& \sigma_{2 *} D_{1} \cdot D_{2}=D_{1} \cdot \sigma_{2}^{*} D_{2}=D_{1} \cdot D_{2}=4, \\
& \sigma_{2 *} D_{1} \cdot D_{3}=D_{1} \cdot \sigma_{2}^{*} D_{3}=D_{1} \cdot D_{3}=1, \\
& \sigma_{2 *} D_{2} \cdot D_{2}=D_{2} \cdot D_{2}=2, \\
& \sigma_{2 *} D_{2} \cdot D_{3}=D_{2} \cdot D_{3}=0, \\
& \sigma_{2 *} D_{3} \cdot D_{3}=D_{3} \cdot D_{3}=-2 .
\end{aligned}
$$

So the only difficult intersection to calculate is $\sigma_{2 *} D_{1} \cdot D_{1}$. We do this by writing $\sigma_{2 *} D_{1}=a D_{1}+b D_{2}+c D_{3}$, and noting $\sigma_{2 *} D_{1} \cdot \sigma_{2 *} D_{1}=D_{1} \cdot D_{1}=2$, so $2 a^{2}+2 b^{2}-$ $2 c^{2}+8 a b+2 a c=2$. Together with the Equations (3) and (4) above, we can solve for $a, b$, and $c$, and find $\sigma_{2 *} D_{1}=-D_{1}+4 D_{2}-D_{3}$.

At this point, it is useful to define the dual basis $\mathcal{D}^{*}$ of $\operatorname{Pic}(V) \otimes Q$. Let us write $[D]_{\mathcal{D}}=[a, b, c]$ if $D=a D_{1}+b D_{2}+c D_{3}$. We define the dual basis $\mathcal{D}^{*}$ such that $[D]_{\mathcal{D}^{*}}=\left[D \cdot D_{1}, D \cdot D_{2}, D \cdot D_{3}\right]$. The change of basis matrix from the basis $\mathcal{D}$ to the basis $\mathcal{D}^{*}$ is given by $Q_{\mathcal{D}}^{-1}$. Hence, in this basis, we get

$$
Q_{\mathcal{D}^{*}}=\left(Q_{\mathcal{D}}^{-1}\right)^{T} Q_{\mathcal{D}} Q_{\mathcal{D}}^{-1}=Q_{\mathcal{D}}^{-1}=\frac{1}{22}\left[\begin{array}{ccc}
-4 & 8 & -2 \\
8 & -5 & 4 \\
-2 & 4 & -12
\end{array}\right],
$$

and by the above calculations,

$$
\left[\sigma_{2 *}\right]_{\mathcal{D}^{*}}=\left[\begin{array}{ccc}
-1 & 4 & -1 \\
0 & 1 & 0 \\
0 & 0 & 1
\end{array}\right] .
$$

The calculation of $\sigma_{1 *}$ is not as easy. We know $\sigma_{1 *} D_{1}=D_{1}$. Let

$$
\begin{aligned}
& \sigma_{1 *} D_{2}=a D_{1}+b D_{2}+c D_{3}, \\
& \sigma_{1 *} D_{3}=d D_{1}+e D_{2}+f D_{3} .
\end{aligned}
$$


We have six unknowns, and the six equations:

$$
\begin{aligned}
& \sigma_{1 *} D_{2} \cdot D_{1}=D_{2} \cdot \sigma_{1}^{*} D_{1}=D_{2} \cdot D_{1}=4, \\
& \sigma_{1 *} D_{3} \cdot D_{1}=D_{3} \cdot \sigma_{1}^{*} D_{1}=D_{3} \cdot D_{1}=1, \\
& \sigma_{1 *} D_{2} \cdot \sigma_{1 *} D_{2}=D_{2} \cdot D_{2}=2, \\
& \sigma_{1 *} D_{3} \cdot \sigma_{1 *} D_{3}=D_{3} \cdot D_{3}=-2, \\
& \sigma_{1 *} D_{2} \cdot \sigma_{1 *} D_{3}=D_{2} \cdot D_{3}=0, \\
& \sigma_{1 *} D_{2} \cdot D_{3}=D_{2} \cdot \sigma_{1}^{*} D_{3} .
\end{aligned}
$$

Solving, we get eight possibilities for $\left[\sigma_{1 *}\right]_{\mathcal{D}^{*}}$. Of these, six have nonintegral entries and one is the identity. Thus, $\left[\sigma_{1 *}\right]_{\mathcal{D}^{*}}$ cannot be any of those, and we get

$$
\left[\sigma_{1 *}\right]_{\mathcal{D}^{*}}=\left[\begin{array}{ccc}
1 & 0 & 0 \\
4 & -1 & 0 \\
1 & 0 & -1
\end{array}\right]
$$

Before continuing, let us introduce a new basis for $\operatorname{Pic}(V)$. Let $H_{2}$ be a hyperplane section that contains $P$. Then $D_{2}=\pi_{2}^{-1} H_{2}$ contains $\pi_{2}^{-1} P=D_{3}$, so has two components. Hence, $B_{2}=D_{2}-D_{3}$ is an effective divisor, generated by an irreducible curve. We also note that $B_{1}=\sigma_{1 *} D_{3}=D_{1}-D_{3}$ is represented by an irreducible -2 curve. Let $\mathcal{B}=\left\{B_{1}, B_{2}, D_{3}\right\}$ be our new basis for $\operatorname{Pic}(V)$. The change of basis matrix from $\mathcal{D}^{*}$ to $\mathcal{B}^{*}$ is given by

$$
S=\left[\begin{array}{ccc}
1 & 0 & -1 \\
0 & 1 & -1 \\
0 & 0 & 1
\end{array}\right]
$$

and

$$
Q_{\mathcal{B}^{*}}=\left(S^{-1}\right)^{T} Q_{\mathcal{D}^{*}} S^{-1}=\frac{1}{22}\left[\begin{array}{ccc}
-4 & 8 & 2 \\
8 & -5 & 7 \\
2 & 7 & -1
\end{array}\right]=Q_{\mathcal{B}}^{-1} .
$$

We also have $\left[\sigma_{i *}\right]_{\mathcal{B}^{*}}=S\left[\sigma_{i *}\right]_{\mathcal{D}^{*}} S^{-1}$, which yields the matrices $T_{1}=\left[\sigma_{1 *}\right]_{\mathcal{B}^{*}}$ and $T_{2}=\left[\sigma_{2 *}\right]_{\mathcal{B}^{*}}$ introduced in Theorem 0.2. This is the basis referred to in that theorem and the one we will use from now on.

We now have two elements $T_{1}$ and $T_{2}$ of the group $\mathcal{O}$. Since $D_{3}$ is a -2 curve, we also have the reflection through $D_{3}$, which yields

$$
R=[R]_{\mathcal{B}^{*}}=\left[\begin{array}{ccc}
1 & 0 & 3 \\
0 & 1 & 2 \\
0 & 0 & -1
\end{array}\right]
$$

There is another element of $\mathcal{O}$, not generated by $T_{1}, T_{2}, R$ and -1 . This is the map $T_{3}$ introduced in Theorem 0.2. This map was found by looking for small divisors with self intersection equal to -2 . Not all are generated by $D_{3}$ and $\left\langle T_{1}, T_{2}, R,-1\right\rangle$. By assuming these divisors can be generated from $D_{3}$ and an element 
of $\mathcal{O}$, we find $T_{3}$. Note that we do not necessarily know that there exists a $\sigma_{3}$ such that $T_{3}=\sigma_{3 *}$.

THEOREM 2.1. The group $\mathcal{O}^{+}$is generated by $T_{1}, T_{2}, T_{3}$, and $R$.

The proof I have is an uninteresting method of descent, so I will spare the reader. For our purposes it is enough to know that the group $\left\langle T_{1}, T_{2}, T_{3}, R\right\rangle$ has finite index in $\mathcal{O}^{+}$, which we will show after this next result.

THEOREM 2.2. The group $\mathcal{O}^{\prime \prime}$ is generated by $T_{1}, T_{2}$, and $T_{3}$.

Proof. It is clear that $T_{1}$ and $T_{2}$ are in $\mathcal{O}^{\prime \prime}$, since they are images of elements of $\mathcal{A}$. To show that $T_{3}$ is in $\mathcal{O}^{\prime \prime}$, it is enough to show that $T_{3}(C)$ is effective for any -2 curve $C$. Let us first note that the divisors $B_{2}$ and $B_{1}+2 B_{2}$ are both in $W$ (just check that their intersections with their irreducible components are nonnegative). Thus, for any effective divisor $E$ with $[E]_{\mathcal{B}^{*}}=[a, b, c]$, we know that $b=B_{2} \cdot E \geqslant 0$ and $a+2 b=\left(B_{1}+2 B_{2}\right) \cdot E \geqslant 0$. Given a -2 curve $C$ with $[C]_{\mathcal{B}^{*}}=[a, b, c]$, we know $b \geqslant 0$ and $a+2 b \geqslant 0$ since $C$ is effective. If $T_{3}(C)$ is not effective then, by RiemannRoch, $-T_{3}(C)$ is effective, so

$$
\begin{aligned}
& B_{2} \cdot\left(-T_{3}(C)\right)=-T_{3} B_{2} \cdot C=-b \geqslant 0, \\
& \left(B_{1}+2 B_{2}\right) \cdot\left(-T_{3}(C)\right)=-T_{3}\left(B_{1}+2 B_{2}\right) \cdot C=-(a+2 b) \geqslant 0 .
\end{aligned}
$$

Thus $a=b=0$, so $-2=C \cdot C=c^{2} / 22$, which has no solutions in Z. Hence, no such $C$ can exist, $T_{3} \in \mathcal{O}^{\prime \prime}$, and $\left\langle T_{1}, T_{2}, T_{3}\right\rangle \leqslant \mathcal{O}^{\prime \prime}$.

Using the relation $T^{-1} R T \in \mathcal{O}^{\prime}$ for any $T \in \mathcal{O}^{+}$, it is easy to show that any $T$ can be decomposed as $T=T^{\prime} R^{\prime}$, where $T^{\prime} \in\left\langle T_{1}, T_{2}, T_{3}\right\rangle$ and $R^{\prime} \in \mathcal{O}^{\prime}$. Thus, $\mathcal{O}^{\prime \prime}=$ $\left\langle T_{1}, T_{2}, T_{3}\right\rangle$, for otherwise, there would be an element in both $\mathcal{O}^{\prime \prime}$ and $\mathcal{O}^{\prime}$.

As mentioned earlier, the action of $\mathcal{O}^{\prime \prime}$ induces a tiling of hyperbolic geometry. A Poincaré disc model of this tiling is shown in Figure 1. This model is found as follows: Since $Q$ is symmetric, there exists a matrix $P$ such that $P^{T}=P^{-1}$ and

$$
P^{T} Q P=\left[\begin{array}{ccc}
-a_{1}^{2} & 0 & 0 \\
0 & -a_{2}^{2} & 0 \\
0 & 0 & a_{3}^{2}
\end{array}\right]=A^{T} J A,
$$

where

$$
A=\left[\begin{array}{ccc}
a_{1} & 0 & 0 \\
0 & a_{2} & 0 \\
0 & 0 & a_{3}
\end{array}\right] \text { and } J=\left[\begin{array}{ccc}
-1 & 0 & 0 \\
0 & -1 & 0 \\
0 & 0 & 1
\end{array}\right] .
$$

Hence, the map $\mathbf{x} \mapsto A P^{T} \mathbf{x}$ sends $\mathcal{H}$ to the canonical Lorentz model $\mathcal{H}_{0}$ of hyperbolic geometry described by the hyperboloid $x^{2}+y^{2}-z^{2}=-1$ and $z>0$. We use stereographic projection through the point $(0,0,-1)$ and onto the plane $z=0$ to map $\mathcal{H}_{0}$ to the Poincare disc (see [5] for more details). We note that $T_{2}$ and $T_{3}$ are reflections 


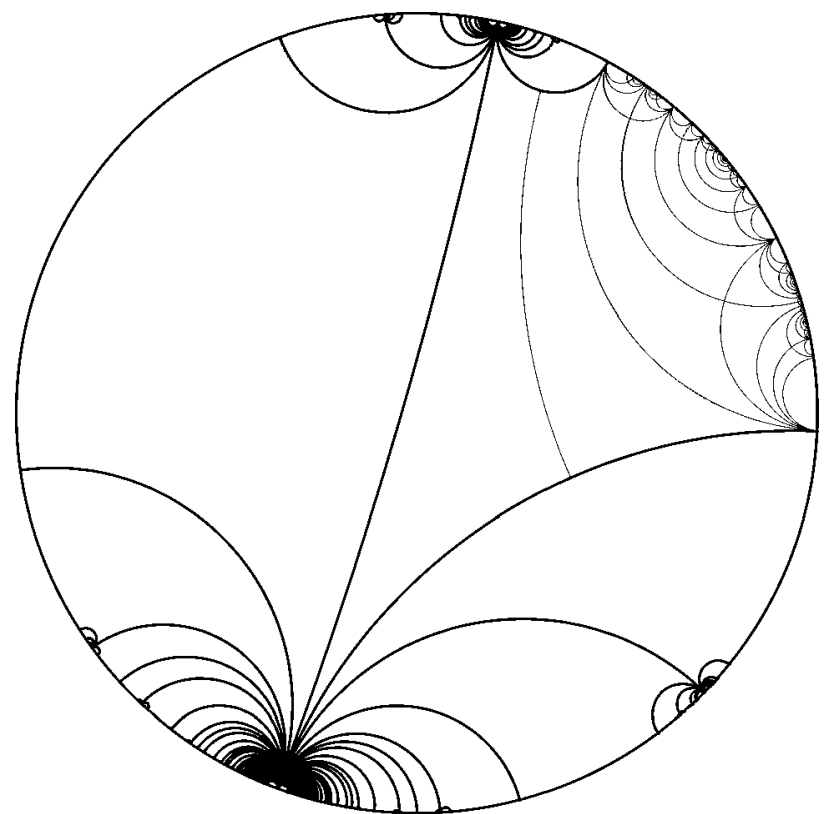

Figure 1. The tiling induced by $\mathcal{O}^{\prime \prime}$ (heavy lines). One of those tiles has been further tiled by $\mathcal{O}^{+}$(light lines). The fundamental domain of $\mathcal{O}^{\prime \prime}$ has infinite area and the fundamental domain of $\mathcal{O}^{+}$has area equal to $\pi$.

(their determinants are -1 ). The boundary of our choice of fundamental domain includes the lines through which $T_{2}$ and $T_{1} T_{3} T_{1}$ reflect, and the line that joins endpoints of the mirrors and includes the center of the rotation $T_{1}$ (note that $T_{1}$ is a rotation through $\pi$ since its determinant is 1 and $T_{1}^{2}=1$ ). In Figure 1, one of the tiles for $\mathcal{O}^{\prime \prime}$ is further tiled by $\mathcal{O}^{+}$. It is evident from the figure that the area of the fundamental domain for $\mathcal{O}^{+}$is $\pi$. For those unconvinced that $\left\langle T_{1}, T_{2}, T_{3}, R\right\rangle$ is all of $\mathcal{O}^{+}$, this observation shows that this group has finite index in $\mathcal{O}^{+}$. We also readily see, from Figure 1 , that $\mathcal{O}^{\prime \prime} \cong Z / 2 Z * Z / 2 Z * Z / 2 Z$.

\section{The Fractal Dimension of $\mathcal{A}$}

In this section, we investigate $N_{\mathcal{O}_{B^{*}}^{\prime}(\mathbf{a})}(t)=\#\left\{\mathbf{x} \in \mathcal{O}_{\mathcal{B}^{*}}^{\prime \prime}(\mathbf{a}): h(\mathbf{x})<t\right\}$. The height we use is $h(\mathbf{x})=x_{1}+x_{2}+2 x_{3}$, which is the height given by $D=D_{1}+D_{2}$. Since we will not again change the basis, let us drop the references to the basis $\mathcal{B}^{*}$. The orbit $\mathcal{O}^{\prime \prime}(\mathbf{a})$ has a natural tree structure. The fractal dimension of similar trees have been investigated by the author [3] in reference to orbits of integer solutions of the Hurwitz equation, and by Boyd [8-10] in reference to the fractal dimension of the Apollonian circle packing. McMullen [15] has also recently developed an algorithm to approximate these types of dimensions. In this section, we use the methods of Boyd and the 
author. Unfortunately, each tree seems to have its own subtle characteristics which make generalized results difficult to extract.

As McMullen points out, these dimensions are also related to the smallest eigenvalue $\lambda_{0}$ for the Laplacian in the hyperbolic space with respect to the fundamental domain of a Fuchsian group (care of the result by Lax and Phillips [14]). For a two dimensional hyperbolic space (such as our case), the relation is $\lambda_{0}=\alpha(1-\alpha)$. Thus, as a corollary of Theorem 0.1 , we get an estimate of the minimal eigenvalue $\lambda_{0}$ of the two dimensional hyperbolic Laplacian with respect to the group $\mathcal{O}^{\prime \prime}$.

A tree is a connected graph with no loops. The group $\mathcal{O}^{\prime \prime}$ may be thought of as a graph whose nodes are elements of the group, and whose edges are pairs of elements $\left(T, T_{i} T\right)$ for every $T \in \mathcal{O}^{\prime \prime}$ and $i=1,2$ or 3 . Since $\mathcal{O}^{\prime \prime} \cong Z / 2 Z * Z / 2 Z * Z / 2 Z, \mathcal{O}^{\prime \prime}$ forms a tree. A subset $U$ of $\mathcal{O}^{\prime \prime}$ is a subtree if it is connected. In this paper, we will further require that every subtree includes the identity.

Since $\mathcal{O}^{\prime \prime} \cong Z_{/} / 2 Z * Z_{/} / 2 Z * Z_{/} / 2 Z$, every element $T \in \mathcal{O}^{\prime \prime}$ has a unique representation of the form $T=T_{k_{m}} \cdots T_{k_{1}}$, where $k_{i} \in\{1,2,3\}$ and $k_{i} \neq k_{i+1}$ for all $i=1,2, \ldots$, $m-1$. Three subtrees of $\mathcal{O}^{\prime \prime}$ which are of particular interest are the subtrees

$$
U_{i}=\left\{T=T_{k_{m}} \cdots T_{k_{1}}: k_{1} \neq i, m \geqslant 0\right\}
$$

The tree structure of $\mathcal{O}^{\prime \prime}$ induces a tree structure on orbits. The forward tree $U^{+}(\mathbf{a})$ is the tree $U^{+}(\mathbf{a})=\left\{T(\mathbf{a}): T \in U_{i}\right\}$ where $i$ is chosen so that $h\left(T_{i}(\mathbf{a})\right)<h(\mathbf{a})$. If no such $i$ exists, then $U^{+}(\mathbf{a})=\mathcal{O}^{\prime \prime}(\mathbf{a})$. We will also set $U_{\mathbf{a}}^{+}=U_{i}$ for the appropriate choice of $i$ depending on a.

We will also be interested in the subtrees $U(\mathbf{a}, y)=\{\mathbf{x} \in U(\mathbf{a}): h(\mathbf{x})<y\}$, where $U$ is a subtree of $\mathcal{O}^{\prime \prime}$. We note that descent, when it occurs, is unique. That is, if $h\left(T_{i} \mathbf{x}\right)<h(\mathbf{x})$, then $h\left(T_{j} \mathbf{x}\right)>h(\mathbf{x})$ for $j \neq i$. Consequently, $U(\mathbf{a}, y)$ is a subtree.

The boundary $\partial U$ of a tree $U$ is the set

$$
\partial U=\left\{T_{i} T: T \in U, T_{i} T \notin U, i=1,2,3\right\} .
$$

The boundary of a subtree of an orbit $\mathcal{O}^{\prime \prime}(\mathbf{a})$ is defined in a similar fashion.

The fundamental object we study to evaluate the asymptotic behavior of $N_{\mathcal{O}^{\prime \prime}(\mathbf{a})}(t)$ is the function $f_{\mathbf{a}}(s)=\sum_{\mathbf{x} \in \mathcal{O}^{\prime \prime}(\mathbf{a})}(h(\mathbf{x}))^{-s}$. There exists a real number $\alpha(\mathbf{a})$ such that $f_{\mathbf{a}}(s)$ converges for all $s>\alpha(\mathbf{a})$, and diverges for all $s<\alpha(\mathbf{a})$. This boundary of convergence $\alpha(\mathbf{a})$ is related to $N_{\mathcal{O}^{\prime \prime}(\mathbf{a})}(t)$ by the following classical result:

$$
\limsup _{t \rightarrow \infty} \frac{\log N_{\mathcal{O}^{\prime \prime}(\mathbf{a})}(t)}{\log t}=\alpha(\mathbf{a}) \text {. }
$$

In the study of $f_{\mathbf{a}}(s)$, we will have occasion to use the following functions:

$$
f_{\mathbf{a}}^{+}(s)=\sum_{\mathbf{x} \in U^{+}(\mathbf{a})}(h(\mathbf{x}))^{-s}, \quad f_{\mathbf{a}}^{+}(s, y)=\sum_{\mathbf{x} \in U^{+}(\mathbf{a}, y)}(h(\mathbf{x}))^{-s} .
$$


Let us also define $N_{\mathbf{a}}^{+}(t)=N_{U^{+}(\mathbf{a})}(t)$.

We make the following definitions because of the results we will find. Let

$$
\begin{aligned}
l_{1}(\mathbf{a}, s)= & \begin{cases}\left(\left(3 a_{1}+2 a_{3}\right) / 8\right)^{-s} & \text { if } a_{1} \geqslant 2 a_{3}, \\
\left(\left(2 a_{1}+3 a_{3}\right) / 7\right)^{-s} & \text { if } a_{1}<2 a_{3},\end{cases} \\
l_{2}(\mathbf{a}, s)= & \sum_{k=0}^{\infty}\left(\left((2 k+3 / 7) a_{1}+\left(11 k^{2}+5 k / 7\right) a_{2}-(k-2 / 7) a_{3}\right)^{-s}+\right. \\
& \left.+\left((2 k+7 / 4) a_{1}+\left(11 k^{2}+(61 k+21) / 4\right) a_{2}-(k+3 / 8) a_{3}\right)^{-s}\right) \\
l_{3}(\mathbf{a}, s)= & \sum_{k=0}^{\infty}\left(\left(-(2 k-1 / 4) a_{1}+\left(11 k^{2}+5 k / 4\right) a_{2}+(k+3 / 8) a_{3}\right)^{-s}+\right. \\
& \left.+\left(-(2 k+3 / 7) a_{1}+\left(11 k^{2}+61 k / 7+12 / 7\right) a_{2}+(k+5 / 7) a_{3}\right)^{-s}\right) \\
l(\mathbf{a}, s)= & l_{i}(\mathbf{a}, s) \quad \text { if } h\left(T_{i} \mathbf{a}\right)<h(\mathbf{a}), \\
L(s, y)= & \sum_{\mathbf{a} \in \partial U^{+}(\mathbf{r}, y)} l(\mathbf{a}, s),
\end{aligned}
$$

where $\mathbf{r}=[2,9,1]$. The choice of $\mathbf{r}=[2,9,1]$ is very particular. We will explain why we make this choice later. Let us also define

$$
\begin{aligned}
& g_{1}(\mathbf{a}, s)= \begin{cases}\left(\left(2 a_{1}+3 a_{3}\right) / 7\right)^{-s} & \text { if } a_{1} \geqslant 2 a_{3}, \\
\left(\left(3 a_{1}+2 a_{3}\right) / 8\right)^{-s} & \text { if } a_{1}<2 a_{3},\end{cases} \\
& g_{2}(\mathbf{a}, s)=\sum_{k=0}^{\infty}\left(\left((2 k+1 / 4) a_{1}+\left(11 k^{2}-5 k / 4\right) a_{2}-(k-3 / 8) a_{3}\right)^{-s}+\right. \\
& \left.+\left((2 k+11 / 7) a_{1}+\left(11 k^{2}+93 k / 7+4\right) a_{2}-(k+2 / 7) a_{3}\right)^{-s}\right), \\
& g_{3}(\mathbf{a}, s)=\sum_{k=0}^{\infty}\left(\left(-(2 k-3 / 7) a_{1}+\left(11 k^{2}-5 k / 7\right) a_{2}+(k+2 / 7) a_{3}\right)^{-s}+\right. \\
& \left.+\left(-(2 k+1 / 4) a_{1}+\left(11 k^{2}+27 k / 4+1\right) a_{2}+(k+5 / 8) a_{3}\right)^{-s}\right), \\
& g(\mathbf{a}, s)=g_{i}(\mathbf{a}, s) \quad \text { if } h\left(T_{i} \mathbf{a}\right)<h(\mathbf{a}), \\
& G(s, y)=\sum_{\mathbf{a} \in \partial U^{+}(\mathbf{r}, y)} g(\mathbf{a}, s) .
\end{aligned}
$$

The first main result that we aim for in this section is

THEOREM 3.1. For $s>1 / 2, x$ much larger than $y$ and $y$ sufficiently large, we have

$$
f_{\mathbf{r}}^{+}(s) \geqslant L(s, y) f_{\mathbf{r}}^{+}(s), \quad f_{\mathbf{r}}^{+}(s, x) \leqslant G(s, y) f_{\mathbf{r}}^{+}(s, x)+\mathrm{O}(y) .
$$

This result can be thought of as a self similarity statement. The following two lemmas are fundamental to finding such a self similarity in the tree $\mathcal{O}^{\prime \prime}(\mathbf{a})$. 
LEMMA 3.2. Suppose $c>0$. Then

$$
\begin{aligned}
& f_{c \mathbf{a}}(s)=c^{-s} f_{\mathbf{a}}(s), \quad f_{c \mathbf{a}}^{+}(s)=c^{-s} f_{\mathbf{a}}^{+}(s), \\
& f_{c \mathbf{a}}^{+}(s, y)=c^{-s} f_{\mathbf{a}}^{+}(s, y / c), \quad N_{c \mathbf{a}}^{+}(t)=N_{\mathbf{a}}^{+}(t / c) .
\end{aligned}
$$

Proof. The group $\mathcal{O}^{\prime \prime}$ is a group of linear operators. Thus,

$$
h(T(c \mathbf{a}))=h(c T(\mathbf{a}))=\operatorname{ch}(T(\mathbf{a})) .
$$

Hence, $c^{-s}$ factors out of the various $f$. The $y / c$ on the right-hand side of the third equality is necessary to make sure the two trees end at the same places.

LEMMA 3.3. Suppose $h\left(T_{i}(\mathbf{a})\right)<h(\mathbf{a}), h\left(T_{i}(\mathbf{b})\right)<h(\mathbf{b}), h(T(\mathbf{c})) \geqslant 0$ for all $T \in U_{\mathbf{a}}^{+}$, and suppose $\mathbf{a}=\mathbf{b}+\mathbf{c}$. Then, for $s>0$,

$$
f_{\mathbf{a}}^{+}(s) \leqslant f_{\mathbf{b}}^{+}(s), \quad f_{\mathbf{a}}^{+}(s, y) \leqslant f_{\mathbf{b}}^{+}(s, y), \quad N_{\mathbf{a}}^{+}(t) \leqslant N_{\mathbf{b}}^{+}(t) .
$$

Proof. Again, we use linearity. Note that

$$
h(T(\mathbf{a}))=h(T(\mathbf{b})+T(\mathbf{c}))=h(T(\mathbf{b}))+h(T(\mathbf{c})) \geqslant h(T(\mathbf{b}))
$$

and make a node-by-node comparison. In the second equation, note that the righthand side may have more terms.

We use these two lemmas to find bounds on $f_{\mathbf{a}}^{+}(s)$ in terms of $f_{\mathbf{r}}^{+}(s)$ where $\mathbf{r}=[2,9,1]$ and for a such that $h\left(T_{1}(\mathbf{a})\right)<h(\mathbf{a})$.

LEMMA 3.4. Let $\mathbf{a}=\left[a_{1}, a_{2}, a_{3}\right]$ and $a_{2}^{\prime}=3 a_{1}-a_{2}+3 a_{3}$. Suppose $a_{i} \geqslant 0, a_{2}^{\prime} \geqslant 0$, and $h(\mathbf{a})>14$. Suppose there exists $j=2$ or 3 such that $0<h\left(T_{j} T_{1} \mathbf{a}\right)<h\left(T_{1} \mathbf{a}\right)<h(\mathbf{a})$. Then,

$$
f_{\mathbf{a}}^{+}(s)>l_{1}(\mathbf{a}, s) f_{\mathbf{r}}^{+}(s), \quad N_{\mathbf{a}}^{+}(t)>N_{\mathbf{a}}^{+}\left(t l_{1}(\mathbf{a}, 1)\right), \quad f_{\mathbf{a}}^{+}(s, y)<g_{1}(\mathbf{a}, s) f_{\mathbf{r}}^{+}(s, y) .
$$

Proof. Note that the forward tree with respect to $\mathbf{a}, \mathbf{r}$ and $[0,1,0]$ are all $U_{1}$. Recall that $B_{1}=[-2,1,3]$ and $D_{3}=[3,2,-2]$ are both effective, so both $h\left(T B_{1}\right) \geqslant 0$ and $h\left(T D_{3}\right) \geqslant 0$ for all $T \in \mathcal{O}^{\prime \prime}$.

Let us write $\mathbf{a}$ as a linear combination of $\mathbf{r}, D_{3}$ and $[0,1,0]$ :

$$
\begin{aligned}
{\left[a_{1}, a_{2}, a_{3}\right]=} & \left(\left(2 a_{1}+3 a_{3}\right) / 7\right)[2,9,1]+\left(\left(a_{1}-2 a_{3}\right) / 7\right)[3,2,-2]+ \\
& +\left(\left(a_{1}-2 a_{3}\right) / 7-a_{2}^{\prime}\right)[0,1,0] .
\end{aligned}
$$

If $j=2$, then $-2 a_{3}+4 a_{2}^{\prime}+a_{1}<0$. Thus, if $a_{1} \geqslant 2 a_{3}$, then $j=3$ and hence, $2 a_{3}+7 a_{2}^{\prime}-a_{1}<0$, so the coefficients of $D_{3}$ and $[0,1,0]$ are positive. Hence, by Lemmas 3.2 and 3.3,

$$
f_{\mathbf{a}}^{+}(s, y) \leqslant c^{-s} f_{\mathbf{r}}^{+}(s, y / c),
$$

where $c=\left(2 a_{1}+3 a_{3}\right) / 7$. Since $h\left(T_{1} \mathbf{a}\right)>0$, we know $5 a_{1}+3 a_{3}>2 a$, so

$$
\frac{49 c}{2}>7 a_{1}+7 a_{3}>2 a_{1}+2 a_{2}+4 a_{3}=2 h(\mathbf{a})>28>49 / 2 .
$$


Thus, $c>1$, and $f_{\mathbf{r}}^{+}(s, y / c)<f_{\mathbf{r}}^{+}(s, y)$, so

$$
f_{\mathbf{a}}^{+}(s, y)<\left(\left(2 a_{1}+3 a_{3}\right) / 7\right)^{-s} f_{\mathbf{r}}^{+}(s, y) .
$$

Suppose now that $a_{1}<2 a_{3}$. Then the coefficient of $[0,1,0]$ is also negative, and again by Lemmas 3.2 and 3.3,

$$
f_{\mathbf{a}}^{+}(s) \geqslant\left(\left(2 a_{1}+3 a_{3}\right) / 7\right)^{-s} f_{\mathbf{r}}^{+}(s), \quad N_{\mathbf{a}}^{+}(t) \geqslant N_{\mathbf{r}}^{+}\left(7 t /\left(2 a_{1}+3 a_{3}\right)\right) .
$$

We can also write

$$
\begin{aligned}
{\left[a_{1}, a_{2}, a_{3}\right]=} & \left(\left(3 a_{1}+2 a_{3}\right) / 8\right)[2,9,1]-\left(\left(a_{1}-2 a_{3}\right) / 8\right)[-2,1,3]+ \\
& +\left(\left(a_{1}-2 a_{3}\right) / 4+a_{2}^{\prime}\right)[0,1,0] .
\end{aligned}
$$

Hence, if $a_{1} \geqslant 2 a_{3}$, then

$$
f_{\mathbf{a}}^{+}(s) \geqslant\left(\left(3 a_{1}+2 a_{3}\right) / 8\right)^{-s} f_{\mathbf{r}}^{+}(s), \quad N_{\mathbf{a}}^{+}(t) \geqslant N_{\mathbf{r}}^{+}\left(\left(8 t /\left(3 a_{1}+2 a_{3}\right)\right) .\right.
$$

If $a_{1} \leqslant 2 a_{3}$, then as before, $\left(a_{1}-2 a_{3}\right) / 4+a_{2}^{\prime}<0$, so

$$
f_{\mathbf{a}}^{+}(s, y) \leqslant\left(\left(3 a_{1}+2 a_{3}\right) / 8\right)^{-s} f_{\mathbf{r}}^{+}(s, y) .
$$

As before, since $h(\mathbf{a})>14$, we have $\left(3 a_{1}+2 a_{3}\right) / 8>1$.

Finding bounds on $f_{\mathbf{a}}^{+}(s)$ for a for which $T_{2}$ or $T_{3}$ give descent is a little more problematic. The main difference is that the products $T_{2} T_{3}$ and $T_{3} T_{2}$ both have eigenvalues of 1 with multiplicity three, so the growth of solutions on the branches generated by $T_{2}$ and $T_{3}$ is very slow. This is why we consider the infinite branch generated by $T_{2}$ and $T_{3}$, and the boundary of this branch.

LEMMA 3.5 .

$$
\begin{aligned}
& T_{1}\left(T_{3} T_{2}\right)^{k}=\left[\begin{array}{ccc}
-4 k & 22 k^{2}+8 k & 2 k+1 \\
-18 k+3 & 99 k^{2}+3 k-1 & 9 k+3 \\
-2 k+1 & 11 k^{2}-7 k & k
\end{array}\right], \\
& T_{1} T_{2}\left(T_{3} T_{2}\right)^{k}=\left[\begin{array}{ccc}
-4 k & 22 k^{2}+8 k & 2 k+1 \\
-18 k-3 & 99 k^{2}+69 k+11 & 9 k+6 \\
-2 k-1 & 11 k^{2}+15 k+4 & k+1
\end{array}\right], \\
& T_{1}\left(T_{2} T_{3}\right)^{k}=\left[\begin{array}{ccc}
4 k & 22 k^{2}-8 k & -2 k+1 \\
18 k+3 & 99 k^{2}-3 k-1 & -9 k+3 \\
2 k+1 & 11 k^{2}+7 k & -k
\end{array}\right], \\
& T_{1} T_{3}\left(T_{2} T_{3}\right)^{k}=\left[\begin{array}{ccc}
4 k+4 & 22 k^{2}+36 k+14 & -2 k-1 \\
18 k+15 & 99 k^{2}+129 k+41 & -9 k-3 \\
2 k+1 & 11 k^{2}+7 k & -k
\end{array}\right] .
\end{aligned}
$$


This can be proved using induction. Note that, for large $k$, the nodes $T_{1}\left(T_{3} T_{2}\right)^{k} \mathbf{a}$, etc., are very close to a multiple of $\mathbf{r}=[2,9,1]$. This explains our choice for $\mathbf{r}$.

LEMMA 3.6. Suppose $s>1 / 2$ and $h\left(T_{i}(\mathbf{a})\right)<h(\mathbf{a})$ for $i=2$ or 3 . Then

$$
\begin{aligned}
& f_{\mathbf{a}}^{+}(s)>l_{i}(\mathbf{a}, s) f_{\mathbf{r}}^{+}(s), \\
& f_{\mathbf{a}}^{+}(s, y)<g_{i}(\mathbf{a}, s) f_{\mathbf{r}}^{+}(s, y)+\mathrm{O}\left(a_{2}^{-s}\right) .
\end{aligned}
$$

Proof. First, suppose $i=2$. For the first inequality,

$$
\begin{aligned}
f_{\mathbf{a}}^{+}(s) & =\sum_{k=0}^{\infty}\left(\left(h\left(\left(T_{2} T_{3}\right)^{k} \mathbf{a}\right)^{-s}+\left(h\left(T_{3}\left(T_{2} T_{3}\right)^{k} \mathbf{a}\right)^{-s}+f_{T_{1}\left(T_{2} T_{3}\right)^{k} \mathbf{a}}^{+}(s)+f_{T_{1} T_{3}\left(T_{2} T_{2}\right)^{k} \mathbf{a}}^{+}(s)\right)\right.\right. \\
& >\sum_{k=0}^{\infty} f_{T_{1}\left(T_{2} T_{3}\right)^{k} \mathbf{a}}^{+}(s)+f_{T_{1} T_{3}\left(T_{2} T_{3}\right)^{k} \mathbf{a}}^{+}(s) .
\end{aligned}
$$

Now apply Lemmas 3.4 and 3.5. For the second inequality,

The sum

$$
\begin{aligned}
f_{\mathbf{a}}^{+}(s, y) \leqslant & \sum_{k=0}^{\infty}\left(\left(h\left(\left(T_{2} T_{3}\right)^{k} \mathbf{a}\right)\right)^{-s}+\left(h\left(T_{3}\left(T_{2} T_{3}\right)^{k} \mathbf{a}\right)\right)^{-s}+\right. \\
& \left.+f_{T_{1}\left(T_{2} T_{3}\right)^{k} \mathbf{a}}^{+}(s, y)+f_{T_{1} T_{3}\left(T_{2} T_{3}\right)^{k} \mathbf{a}}^{+}(s, y)\right) .
\end{aligned}
$$

$$
\sum_{k=0}^{\infty} f_{T_{1}\left(T_{2} T_{3}\right)^{k} \mathbf{a}}^{+}(s, y)+f_{T_{1} T_{3}\left(T_{2} T_{3}\right)^{k} \mathbf{a}}^{+}(s, y)
$$

is estimated using Lemmas 3.4 and 3.5, and gives us $g_{2}(\mathbf{a}, s)$. For the sum

$$
\sum_{k=0}^{\infty}\left(h\left(\left(T_{2} T_{3}\right)^{k} \mathbf{a}\right)\right)^{-s}+\left(h\left(T_{3}\left(T_{2} T_{3}\right)^{k} \mathbf{a}\right)\right)^{-s},
$$

we look at $h\left(\left(T_{2} T_{3}\right)^{k} \mathbf{a}\right)^{-s}$, and leave the other term to the reader. Note that

$$
\begin{aligned}
\left(h\left(\left(T_{2} T_{3}\right)^{k} \mathbf{a}\right)\right)^{-s}= & \left(h \left(\left[-(2 k-1) a_{1}+\left(11 k^{2}-7 k\right) a_{2}+k a_{3}, a_{2},-\right.\right.\right. \\
& \left.\left.\left.-4 k a_{1}+\left(22 k^{2}+8 k\right) a_{2}+(2 k+1) a_{3}\right]\right)\right)^{-s} \\
= & \left(55 a_{2} k^{2}+\left(-10 a_{1}+9 a_{2}+5 a_{3}\right) k+\left(a_{1}+a_{2}+2 a_{3}\right)\right)^{-s} .
\end{aligned}
$$

Since $T_{2}$ gives descent, we know $4 a_{1}+a_{3}<2 a_{2}$, so each coefficient is positive. Thus,

$$
\sum_{k=0}^{\infty}\left(h\left(\left(T_{2} T_{3}\right)^{k} \mathbf{a}\right)\right)^{-s}<\sum_{k=0}^{\infty}\left(55 a_{2} k^{2}\right)^{-s}=\mathrm{O}\left(a_{2}^{-s}\right),
$$

since $s>1 / 2$. The other term is similar, as are the arguments for $i=3$.

We are now ready to prove Theorem 3.1:

Proof of Theorem 3.1. For $y$ sufficiently large, we can write

$$
f_{\mathbf{r}}^{+}(s)=f_{\mathbf{r}}^{+}(s, y)+\sum_{\mathbf{a} \in \partial U^{+}(\mathbf{r}, y)} l(\mathbf{a}, s) f_{\mathbf{a}}^{+}(s)>L(s, y) f_{\mathbf{r}}^{+}(s) .
$$


For $x$ much larger than $y$, we can also write

$$
f_{\mathbf{r}}^{+}(s, x) \leqslant f_{\mathbf{r}}^{+}(s, y)+\sum_{\mathbf{a} \in \partial U^{+}(\mathbf{r}, y)}\left(g(\mathbf{a}, s) f_{\mathbf{r}}^{+}(s, x)+\mathrm{O}\left(a_{2}^{-s}\right)\right) .
$$

Note that $a_{2} \geqslant 9$, and by Theorem 0.3 , there are $\mathrm{O}(y)$ terms in the sum over $U^{+}(\mathbf{r}, y)$. There are also $\mathrm{O}(y)$ terms in the sum $f_{\mathbf{r}}^{+}(s, y)$, each of which is less than one. Thus, $f_{\mathbf{r}}^{+}(s, x) \leqslant G(s, y) f_{\mathbf{r}}^{+}(s, x)+\mathrm{O}(y)$.

COROLLARY 3.7. Suppose $s_{1}>0$ and $s_{2}>0$, both depending on $y$, are chosen such that $L\left(s_{1}, y\right)=U\left(s_{2}, y\right)=1$. Then $s_{1} \leqslant \alpha(\mathbf{r}) \leqslant s_{2}$.

Proof. From Theorem 3.1, we have $f_{\mathbf{r}}^{+}(s)>L(s, y) f_{\mathbf{r}}^{+}(s)$. Note that $L(s, y)$ is a decreasing function in $s$. Thus, if $s<s_{1}$, then $L(s, y)>1$. But if $f_{\mathbf{r}}^{+}$converges at $s$, then we can divide both sides above by $f_{\mathbf{r}}^{+}(s)$, which yields the contradiction $1>L(s, y)$. Thus, $f_{\mathrm{r}}^{+}$cannot converge at $s$ for any $s<s_{1}$. Note that $T_{2} B_{2}=T_{3} B_{2}=B_{2}$ and $T_{1}[2,9,1]=[1,0,2]=B_{2}$, so $f_{\mathbf{r}}(s)=5^{-s}+f_{\mathbf{r}}^{+}(s)$. Thus, $f_{\mathbf{r}}(s)$ cannot converge at $s$ for any $s<s_{1}$, either. Thus, $s_{1} \leqslant \alpha(\mathbf{r})$.

For the upper bound, we have, from Theorem 3.1,

$$
f_{\mathbf{r}}^{+}(s, x)<G(s, y) f_{\mathbf{r}}^{+}(s, x)+\mathrm{O}(y) .
$$

Suppose we fix $s<\alpha(\mathbf{r})$. Then $f_{\mathbf{r}}^{+}$does not converge at $s$, so we can choose $x$ so that $f_{\mathbf{r}}^{+}(s, x)$ is as large as we like. Dividing through by $f_{\mathbf{r}}^{+}(s, x)$, we get $1 \leqslant G(s, y)$, so $s \leqslant s_{2}$. Since this is true for all $s<\alpha(\mathbf{r})$, we get $\alpha(\mathbf{r}) \leqslant s_{2}$.

It is easy to write a program to estimate $L(s, y)$ and $G(s, y)$ for any $s$ and $y$. This is how the bounds for $\alpha$ are found. We give some technical details and results in the following section. It is worthwhile noting that this algorithm converges (albeit extremely slowly):

THEOREM 3.8. Let $s_{1}$ and $s_{2}$ be the positive solutions to $L\left(s_{1}, y\right)=G\left(s_{2}, y\right)=1$. Then, for sufficiently large $y$,

$$
0<s_{2}-s_{1}<\frac{5}{7 \log (y / 13)} .
$$

Proof. Let us look at $l_{1}(\mathbf{a}, s)$ and $g_{1}(\mathbf{a}, s)$. If $a_{1} \geqslant 2 a_{3}$, then

$$
\left(3 a_{1}+2 a_{3}\right) / 8>\left(2 a_{1}+3 a_{3}\right) / 7>(16 / 21)\left(3 a_{1}+2 a_{3}\right) / 8 .
$$

If $a_{1}<2 a_{3}$, then

$$
\left(2 a_{1}+3 a_{3}\right) / 7>\left(3 a_{1}+2 a_{3}\right) / 8>(7 / 12)\left(3 a_{1}+2 a_{3}\right) / 8 .
$$

Thus, $l_{1}(\mathbf{a}, s)<g_{1}(\mathbf{a}, s)<(12 / 7)^{s} l_{1}(\mathbf{a}, s)$. Note that $s_{2} \leqslant 1$ (by Theorem 0.3 ), and for $s \leqslant 1$, we have

$$
g_{1}(\mathbf{a}, s)<(12 / 7) l_{1}(\mathbf{a}, s), \quad G(s, y)<(12 / 7) L(s, y) .
$$


Now, suppose $h(\mathbf{a})>y$. Since the components of $T_{1} \mathbf{a}$ are nonnegative for all $\mathbf{a} \in \mathcal{O}^{\prime \prime}(\mathbf{r})$, we know $3 a_{1}-a_{2}+3 a_{3} \geqslant 0$. If $a_{1} \geqslant 2 a_{3}$, then

$$
\left(3 a_{1}+2 a_{3}\right) / 8 \geqslant\left(8 a_{1}+24 a_{1}+14 a_{3}+10 a_{3}+16 a_{3}\right) /(8 \cdot 13)>y / 13 .
$$

If $a_{1}<2 a_{3}$, then

$$
\left(2 a_{1}+3 a_{3}\right) / 7 \geqslant\left(7 a_{1}+19 a_{1}+2 a_{1}+21 a_{3}+14 a_{3} /(7 \cdot 13)>y / 13 .\right.
$$

Thus, $l_{1}(\mathbf{a}, 1)<13 / y$.

By the mean value theorem, there exists an $s$ between $s_{1}$ and $s_{2}$ so that

$$
\frac{L\left(s_{2}, y\right)-L\left(s_{1}, y\right)}{s_{2}-s_{1}}=\frac{\partial L(s, y)}{\partial s} .
$$

Differentiating the expression for $L(s, y)$ with respect to $s$, we get

$$
\begin{aligned}
& L(s, y)=\sum_{\mathbf{a} \in \partial U^{*}(y)} l_{1}(\mathbf{a}, s)=\sum_{\mathbf{a} \in \partial U^{*}(y)} l_{1}(\mathbf{a}, 1)^{s}, \\
& \frac{\partial L(s, y)}{\partial s}=\sum_{\mathbf{a} \in \partial U^{*}(y)} \log \left(l_{1}(\mathbf{a}, 1)\right) l_{1}(\mathbf{a}, 1)^{s} \leqslant-\log (y / 13) L(s, y) .
\end{aligned}
$$

Since $s<s_{2}$, we have $L(s, y)>L\left(s_{2}, y\right)>(7 / 12) G\left(s_{2}, y\right)=7 / 12$. Thus,

$$
\frac{(7 / 12)-1}{s_{2}-s_{1}} \leqslant-(7 / 12) \log (y / 13)
$$

Rearranging gives us the desired result.

A consequence of finding a lower bound $s_{1}$ on the limit supremum $\alpha(\mathbf{r})$ is that it is, in fact, a lower bound on the limit infimum. The key ingredient is Theorem 3.1 of [3], a result that I first saw in Boyd [10]. I will leave the details of its application to this case to the reader. Using this and the previous result, we conclude that the limit exists.

There are a couple more results contained in the statement of Theorem 0.1. We must show that $\alpha(\mathbf{a})$ is independent of $\mathbf{a}$ :

THEOREM 3.9. Suppose the components of $\mathbf{b}$ are positive for all but finitely many $\mathbf{b} \in \mathcal{O}^{\prime \prime}(\mathbf{a})$. Then $\alpha(\mathbf{a})=\alpha(\mathbf{r})$.

Proof. For $y$ sufficiently large, we can write

$$
f_{\mathbf{a}}(s)=f_{\mathbf{a}}(s, y)+\sum_{\mathbf{b} \in \partial U(\mathbf{a}, y)} f_{\mathbf{b}}^{+}(s) \geqslant \sum_{\mathbf{b} \in \partial U(\mathbf{a}, y)} l(\mathbf{b}, s) f_{\mathbf{r}}^{+}(s) .
$$

If $s<\alpha(\mathbf{r})$, then $f_{\mathbf{r}}^{+}(s)$ diverges, so $f_{\mathbf{a}}(s)$ also diverges. Thus, $s<\alpha(\mathbf{a})$. Since this is true for all $s<\alpha(\mathbf{r})$, we get $\alpha(\mathbf{a}) \geqslant \alpha(\mathbf{r})$. But we can also write

$$
f_{\mathbf{a}}(s, x)=f_{\mathbf{a}}(s, y)+\sum_{\mathbf{b} \in \partial U(\mathbf{a}, y)} f_{\mathbf{b}}^{+}(s, x) \leqslant \sum_{\mathbf{b} \in \partial U(\mathbf{a}, y)} g(\mathbf{b}, s) f_{\mathbf{r}}^{+}(s, x)+\mathrm{O}(y),
$$

where $y$ is large enough so that $U(\mathbf{a}, y)$ includes the finitely many elements referred to in the statement of the theorem, and large enough to accommodate Lemma 3.4. 
Suppose $\alpha(\mathbf{a}) \neq \alpha(\mathbf{r})$. Then we can choose $s$ such that $\alpha(\mathbf{r})<s<\alpha(\mathbf{a})$. But then, $f_{\mathbf{r}}^{+}(s)$ converges, so $f_{\mathbf{r}}^{+}(s, x)$ is bounded independent of $x$. Hence, the right-hand side is bounded for all $x$, while the left hand side is not. Thus, we must have $\alpha(\mathbf{a})=\alpha(\mathbf{r})$.

Note that, if $C_{0}$ is a curve, then the components of $\left[C_{\mathcal{B}^{*}}\right.$ are positive for all but finitely many $C \in \mathcal{A}\left(C_{0}\right)$. Thus, $\alpha(C)=\alpha(\mathbf{r})$ for all curves $C$. So let us define $\alpha=\alpha(\mathbf{r})$. We have so far shown that

$$
\lim _{t \rightarrow \infty} \frac{\log \left(N_{\mathcal{A}(C)}(t, D)\right)}{\log t}=\lim _{t \rightarrow \infty} \frac{\log \left(N_{\mathcal{O}^{\prime \prime}(\mathbf{r})}(t)\right)}{\log t}=\alpha
$$

exists and does not depend on the choice of curve $C$. Let us finally establish that this limit is also independent of our choice of ample divisor $D$. I am indebted to the referee for pointing out this result.

THEOREM 3.10 Let $D^{\prime}$ be an arbitrary ample divisor in $\operatorname{Pic}(V)$. Then

$$
\lim _{t \rightarrow \infty} \frac{N_{\mathcal{A}(C)}\left(t, D^{\prime}\right)}{\log t}=\alpha
$$

Proof. Since $D$ and $D^{\prime}$ are ample, there exists an integer $n$ such that $n D^{\prime}-D$ is ample. Thus, for any curve $C$, we know $\left(n D^{\prime}-D\right) \cdot C \geqslant 0$ so $n D^{\prime} \cdot C \geqslant D \cdot C$. Thus,

$$
N_{\mathcal{A}\left(C_{0}\right)}\left(t, D^{\prime}\right)=N_{\mathcal{A}\left(C_{0}\right)}\left(n t, n D^{\prime}\right) \leqslant N_{\mathcal{A}\left(C_{0}\right)}(n t, D),
$$

so

$$
\begin{aligned}
\limsup _{t \rightarrow \infty} \frac{\log N_{\mathcal{A}(C)}\left(t, D^{\prime}\right)}{\log t} & \leqslant \lim _{t \rightarrow \infty} \frac{\log N_{\mathcal{A}(C)}(n t, D)}{\log t} \\
& \leqslant \lim _{t \rightarrow \infty} \frac{\log N_{\mathcal{A}(C)}(t, D)}{\log (t / n)}=\alpha .
\end{aligned}
$$

The other direction is established using a similar argument and the existence of an $m$ such that $m D-D^{\prime}$ is ample.

\section{Technical Details}

To find the bounds on $\alpha$, we must be able to estimate $L(s, y)$ and $G(s, y)$. The evaluation is straight forward, except that we must be able to evaluate sums of the form $S=\sum_{k=0}^{\infty}\left(A k^{2}+B k+C\right)^{-s}$ for $A, B$ and $C \geqslant 0$. Let us write $f(x)=A x^{2}+B x+C$,

$$
S(m)=\sum_{k=m}^{\infty}(f(k))^{-s} \quad \text { and } \quad S=\sum_{k=0}^{m-1}(f(k))^{-s}+S(m) .
$$

We use Euler-Maclaurin summation (see, for example, [12]) to get the estimates

$$
S(m)=\int_{m}^{\infty}(f(x))^{-s} \mathrm{~d} x+\frac{(f(m))^{-s}}{2}+\frac{s(f(m))^{-s-1} f^{\prime}(m)}{12}+R(m, s),
$$


where $R(m, s)<0$ and is bounded below by

$$
-\frac{(f(m))^{-s-3}\left(f^{\prime}(m)\right)^{3}\left(s^{3}+3 s^{2}+2 s\right)}{720}+\frac{(f(m))^{-s-2} f^{\prime}(m) A\left(s^{2}+s\right)}{120} .
$$

If $B^{2}-4 A C \geqslant 0$, then we can estimate the integral with

$$
\frac{(f(m))^{-s+1 / 2}}{\sqrt{A}(2 s-1)} \leqslant \int_{m}^{\infty}(f(x))^{-s} \mathrm{~d} x \leqslant \frac{2(4 A)^{s-1}\left(f^{\prime}(m)\right)^{-2 s+1}}{2 s-1} .
$$

Otherwise $B^{2}-4 A C \leqslant 0$ and

$$
\frac{2(4 A)^{s-1}\left(f^{\prime}(m)\right)^{-2 s+1}}{2 s-1} \leqslant \int_{m}^{\infty}(f(x))^{-s} \mathrm{~d} x \leqslant \frac{(f(m))^{-s+1 / 2}}{\sqrt{A}(2 s-1)} .
$$

Using $m=1$, our approximation for $L\left(.6515,10^{7}\right)$ is -.001378 and our approximation for $G\left(.6538,10^{7}\right)$ is .001797 .

\section{Concluding Remarks}

Let us elaborate on thoughts introduced in the introduction. As mentioned there, part of my interest in the quantity $N_{\mathcal{A}(C)}(t)$ is its presumed relation to the more arithmetic quantity

$$
N_{\mathcal{A}(P)}(t)=\#\left\{Q \in \mathcal{A}(P): h_{D}(Q)<t\right\} .
$$

It would not surprise me if for any K3 surface $V$, generic point $P \in V$, and curve $C \in V$, we have $N_{\mathcal{A}(P)}(t) \gg \ll N_{\mathcal{A}(C)}(t)$. Besides the evidence remarked upon in the introduction, we also note the following: Let $\mathcal{D}=\left\{D_{1}, \ldots, D_{n}\right\}$ be a basis for $\operatorname{Pic}(V)$, and let $D=a_{1} D_{1}+\cdots+a_{n} D_{n}$ be an ample divisor. Let $\mathbf{h}_{\mathcal{D}}(P)=$ $\left[h_{D_{1}}(P), \ldots, h_{D_{n}}(P)\right]$. For any $\sigma \in \mathcal{A}$, we know [13] that $\mathbf{h}_{\mathcal{D}}(\sigma P)=\sigma^{*} \mathbf{h}_{\mathcal{D}}(P)+\mathbf{O}(1)$. The error $\mathbf{O}(1)$ depends on $\sigma$, but not on $P$. If we ignore the error term, then we get the desired result.

If $\mathcal{O}^{\prime \prime}$ is finitely generated, then we can express the error in terms of a finite set of bounds. Unfortunately, since some elements of $\mathcal{O}^{\prime \prime}$ have infinite order and eigenvalues of one, the error may dominate the action. In [2], we are able to significantly improve on the error term because we know how the generators of $\mathcal{A}$ act on $V$. For the $\mathrm{K} 3$ surfaces of this paper, we are at a loss, since we do not know of an automorphism $\sigma_{3}$ that gives $\sigma_{3}^{*}=T_{3}$, if such an automorphism even exists (the image of $\mathcal{A}$ may not include $T_{3}$ ). I would therefore be very interested in any example of a Wehler K3 surface $V$ with Picard number 3 for which one knows a complete description of the generators of $\mathcal{A}$ (or a subgroup of $\mathcal{A}$ with finite index in $\mathcal{A}$ ).

The absence of an error term for the height $h_{D}(C)=D \cdot C$ is essentially because we are working in a local ring. The error for heights over number fields are introduced because of the Archimedean valuations, and because of the finite number of bad primes, where reduction by $p$ gives a different Picard group. 


\section{References}

1. Baragar, A.: Canonical vector heights on algebraic K3 surfaces with Picard number two, Canad. Math. Bull. to appear.

2. Baragar, A.: Rational points on $\mathrm{K} 3$ surfaces in $\mathrm{P}^{1} \times \mathrm{P}^{1} \times \mathrm{P}^{1}$, Math. Ann. 305 (1996), 541-558.

3. Baragar, A.: The exponent for the Markhoff-Hurwitz equations, Pacific J. Math. 182(1) (1998), 1-21.

4. Baragar, A.: Rational curves with zero self intersection on certain K3 surfaces, In: Centre de recherches mathématiques, CRM Proc. Lecture Notes 19, Amer. Math. Soc., Providence, 1999, pp. 1-6.

5. Baragar, A.: A Survey of Classical and Modern Geometries, Prentice-Hall, Upper Saddle River, NJ, 2001.

6. Batyrev, V. V. and Manin, Y. I.: Sur le nombre des points rationnels de hauteur borné des varieétés algébriques., Math. Ann. 286 (1990), 27-43.

7. Billard, H.: Propriétés arithmétiques d'une famille de surfaces K3. Compositio Math. 108(3) (1997), 247-275.

8. Boyd, D. W.: The disk-packing constant, Aequationes Math. 7(2/3) (1972), 182-193.

9. Boyd, D. W.: Improved bounds for the disk-packing constant, Aequationes Math. 9(1) (1973), 99-106.

10. Boyd, D. W.: The sequence of radii of the Apollonian packing, Math. Comp. 39(159) (1982), 249-254.

11. Hartshorne, R.: Algebraic Geometry, Springer-Verlag, New York, 1977.

12. Knopp, K.: Theory and Applications of Infinite Series, Dover, New York, 1990.

13. Lang, S.: Number Theory III, Springer-Verlag, New York, 1991.

14. Lax, P. D. and Phillips, R. S.: The asymptotic distribution of lattice points in Euclidean and non-Euclidean spaces, J. Funct. Anal. 46(3) (1982), 280-350.

15. McMullen, C. T.: Hausdorff dimension and conformal dynamics III: Computation of dimension, Amer. J. Math. 120(4) (1998), 691-721.

16. Pjateckǐ̌-S̆apiro, I. I. and S̈afarevič, I. R.: Torelli theorem for algebraic surfaces of type K3, Nauk SSSR Ser. Mat. 35 (1971), 530-572.

17. Raghunatan, M. S.: Discrete Subgroups of Lie Groups, Springer-Verlag, New York, 1968.

18. Ratcliff, J. S.: Foundations of Hyperbolic Manifolds, Springer-Verlag, New York, 1994.

19. Silverman, J. H.: Rational points on K3 surfaces: A new canonical height, Invent. Math. 105 (1991), 347-373.

20. Wehler, J.: K3-surfaces with Picard number 2, Arch. Math. (Basel) 50(1) (1988), 73-82. 